\title{
Cateterismo Intervencionista na Estenose Valvar Pulmonar Crítica do Recém-Nascido e na Atresia Pulmonar com Septo Interventricular Íntegro: 13 Anos de Experiência de um Serviço Terciário
}

\author{
João Luiz Manica ${ }^{1}$, André Bodini' ${ }^{1}$, Monica Scott Borges ${ }^{1}$, \\ Paulo Renato Mercio Machado', Raul Ivo Rossi Filho'
}

\begin{abstract}
RESUMO
Introdução: A abordagem percutânea é opção de escolha em neonatos portadores de estenose pulmonar crítica (EP) e atresia pulmonar com septo interventricular íntegro (APSI). Neste trabalho são descritos casos tratados consecutivamente em centro de referência e seu seguimento a médio prazo. Métodos: A maioria dos pacientes recebeu infusão endovenosa de prostaglandina pré-procedimento, independentemente da necessidade hemodinâmica. Na abordagem da APSI, foi utilizada guia de ponta rígida e, mais recentemente, valvotomia com cateter de radiofrequência. Na maioria dos casos, foi realizada dilatação sequencial com balão até atingir $110 \%$ a $120 \%$ do diâmetro do anel valvar pulmonar. Resultados: Entre 1998 e 2011, 17 neonatos com APSI (idade mediana de 5 dias, peso de 3,1 $\pm 0,6 \mathrm{~kg}$ ) e 30 neonatos com EP (idade mediana de 12 dias, peso médio de $3 \pm 1,4 \mathrm{~kg}$ ) foram tratados em um serviço terciário. Na APSI, 14 pacientes foram abordados com guia rígida, com sucesso de 71,4\%, e 3 com radiofrequência, com sucesso de $100 \%$. Na EP, o sucesso foi alcançado em todos os casos. O óbito hospitalar foi de $23,5 \%$ no grupo com APSI e de $3,3 \%$ no grupo com EP, nenhum relacionado ao procedimento percutâneo. Na evolução a médio prazo, a taxa de reintervenção por reestenose foi de $21,4 \%$ no grupo com APSI e de $10 \%$ no grupo com EP. Conclusões: A valvoplastia pulmonar na EP e na APSI apresenta resultados clínicos e hemodinâmicos aceitáveis, desde que se observem características anatômicas favoráveis e se mantenha a patência do fluxo pulmonar até o procedimento. A morbidade e a mortalidade dos portadores de EP crítica são mais baixas que as de portadores de APSI.
\end{abstract}

DESCRITORES: Cateterismo. Estenose da valva pulmonar. Atresia pulmonar. Recém-nascido.

\section{ABSTRACT \\ Interventional Catheterization in Neonatal Critical Pulmonary Valve Stenosis and Pulmonary Atresia with Intact Ventricular Septum: 13 Years of Experience at a Tertiary Service}

Background: The percutaneous approach is the therapy of choice in neonates with critical pulmonary stenosis (PS) and pulmonary atresia with intact interventricular septum (PAIVS). Consecutive cases treated at a reference center and their midterm follow-up was reported in this study. Methods: Most of the patients received an intravenous infusion of prostaglandin preoperatively, regardless of the need. For the PAIVS approach, a stiff-tip guidewire was used and more recently, radiofrequency valvotomy. In most cases, sequential balloon dilation was performed until $110 \%$ to $120 \%$ of the pulmonary valve ring diameter was reached. Results: Between 1998 and 2011, 17 neonates with PAIVS (median age of 5 days, mean weight of $3.1 \pm 0.6 \mathrm{~kg}$ ) and 30 neonates with PS (median age of 12 days, mean weight of $3 \pm 1.4 \mathrm{~kg}$ ) were treated at a tertiary center. In PAIVS patients, a stiff-tip guidewire was used in 14 cases, with a success rate of $71.4 \%$, and radiofrequency perforation in 3 cases, with a success rate of $100 \%$. In PS patients, success was achieved in all of the cases. Hospital death was $23.5 \%$ for the PAIVS group and $3.3 \%$ for the PS group, none related to the percutaneous procedure. In the midterm follow-up, the reintervention rate due to restenosis was $21.4 \%$ in the PAIVS group and $10 \%$ in the PS group. Conclusions: Pulmonary valvuloplasty in PS or PAIVS has acceptable clinical and hemodynamic results, as long as favorable anatomic characteristics are observed and patent pulmonary flow is maintained until the procedure. Morbidity and mortality of patients with critical PS are lower than patients with PAIVS.

KEY-WORDS: Catheterization. Pulmonary valve stenosis. Pulmonary atresia. Infant, newborn.

\footnotetext{
1 Instituto de Cardiologia do Rio Grande do Sul - Fundação Universitária de Cardiologia (IC-FUC) - Porto Alegre, RS, Brasil. Correspondência: João Luiz Manica. Av. Princesa Isabel, 370 Santana - Porto Alegre, RS, Brasil - CEP 90620-000 E-mail: digitalizacao.pesquisa@cardiologia.org.br Recebido em: 4/9/2011 • Aceito em: 30/11/2011
} 
A estenose valvar pulmonar crítica do recém-nascido e a atresia pulmonar com septo interventricular íntegro apresentam-se na maioria das vezes com quadro clínico semelhante e representam um capítulo à parte na população pediátrica pela dramaticidade e rápida deterioração clínica. O diagnóstico e o tratamento, quando precoces, estão associados a maior sobrevida; porém, quando realizados na fase tardia, a morbidade e a mortalidade são elevadas. ${ }^{1}$ A valvotomia cirúrgica e o shunt sistêmico-pulmonar eram as únicas opções terapêuticas até meados da década de $1980 .^{2}$ A abordagem percutânea com abertura da valva pulmonar por balão, reconhecidamente, tornou-se opção também em neonatos, não só pela segurança e viabilidade como também pelos bons resultados a longo prazo no que tange tanto ao crescimento ventricular direito e anel valvar pulmonar como às baixas taxas de reintervenção. ${ }^{3}$

O presente estudo tem por objetivo descrever os casos de estenose pulmonar crítica e atresia pulmonar com septo interventricular íntegro tratados consecutivamente, no período neonatal, no Serviço de Hemodinâmica e Cardiologia Intervencionista Pediátrica do Instituto de Cardiologia do Rio Grande do Sul e sua evolução a médio prazo.

\section{MÉTODOS}

Entre janeiro de 1998 e janeiro de 2011, 17 neonatos com diagnóstico de atresia pulmonar com septo interventricular íntegro e 30 com diagnóstico de estenose pulmonar crítica foram submetidos a tratamento percutâneo como primeira abordagem terapêutica em nossa instituição. A decisão inicial pela abordagem biventricular foi baseada na presença de ventrículo direito com anatomia favorável ao ecocardiograma bidimensional com Doppler a cores e na ausência de circulação coronária dependente do ventrículo direito à ventriculografia direita. Os prontuários médicos foram retrospectivamente revisados desde a admissão até a última consulta ambulatorial disponível.
Consentimento informado foi assinado pelos responsáveis dos pacientes submetidos ao procedimento.

\section{Dados ecocardiográficos}

Dados morfológicos do ventrículo direito e da valva pulmonar, além de gradiente transvalvar pulmonar (gradiente VD-AP), foram observados ao ecocardiograma bidimensional com Doppler em cores, bem como a competência e o diâmetro da valva atrioventricular direita, a patência do canal arterial e os defeitos associados.

\section{Dados hemodinâmicos}

Todos os pacientes foram abordados no Laboratório de Hemodinâmica sob anestesia geral. O acesso vascular de eleição foi a veia femoral por punção, realizado em 45 pacientes (95,8\%). Em 2 pacientes $(4,3 \%)$ houve necessidade de flebotomia da veia femoral. Infusão endovenosa de prostaglandina foi utilizada na maior parte dos casos, independentemente da necessidade hemodinâmica, para melhor suporte da guia durante valvoplastia com balão.

Cateterismo direito e ventriculografia direita nas projeções ântero-posterior cranial e lateral com cateter angiográfico Berman (Harmac Medical, Buffalo, Estados Unidos) foram realizados para confirmação diagnóstica, exclusão de circulação coronária dependente do ventrículo direito e mensuração do anel valvar pulmonar.

O cateter angiográfico era, então, substituído pelo cateter de Judkins de coronária direita (Optitorque ${ }^{\mathrm{TM}}$, Terumo, Tóquio, Japão) posicionado na via de saída do ventrículo direito para atravessar a valva pulmonar, geralmente com guia 0,014 polegada nos casos de estenose pulmonar crítica. Nos casos de atresia pulmonar com septo interventricular íntegro, inicialmente os pacientes eram submetidos a tentativa de perfuração da valva pulmonar com guia de ponta rígida. Mais recentemente, quando disponível, os pacientes foram submetidos a valvotomia com cateter de radiofrequência (Baylis Medical, Mississauga, Canadá), conforme técnica previamente estabelecida ${ }^{4}$ (Figura 1). Na maioria
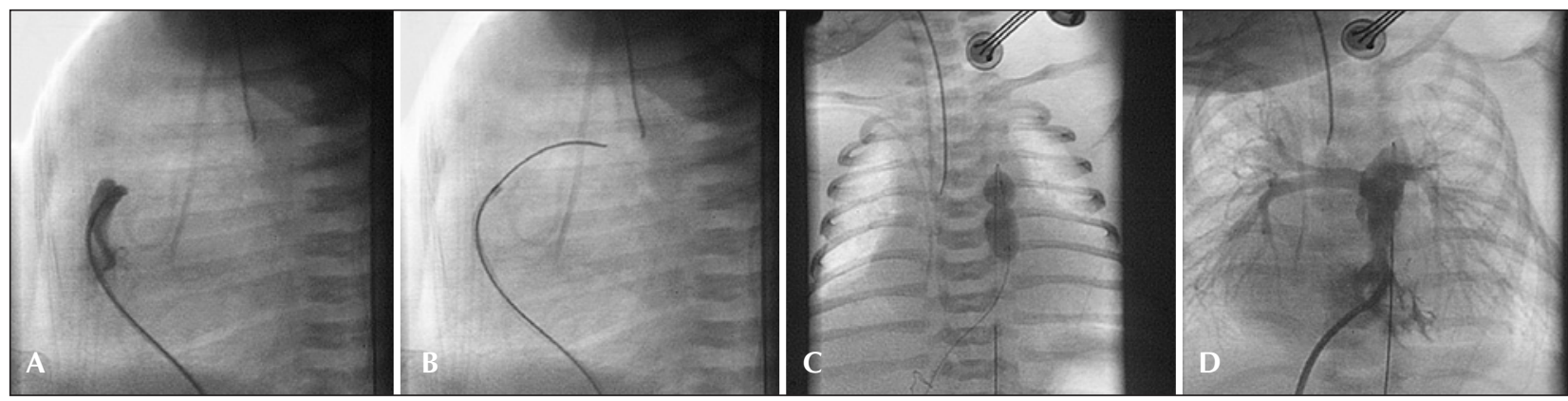

Figura 1 - Em A, injeção manual de contraste em projeção lateral com cateter de coronária direita, demonstrando ausência de fluxo anterógrado através da valva pulmonar. Em B, ainda em projeção lateral, perfuração da valva pulmonar com cateter de radiofrequência. Em C, valvoplastia pulmonar com balão em projeção ântero-superior cranial. Em D, ventriculografia de controle em projeção ântero-superior cranial, demonstrando adequada abertura da valva pulmonar. 
dos casos, foi realizada dilatação sequencial com balão de coronária até atingir diâmetro adequado, em torno de $110 \%$ a $120 \%$ do diâmetro do anel valvar pulmonar. Por fim, foi realizado novo estudo manométrico do lado direito do coração e nova ventriculografia em projeções ântero-posterior cranial e lateral.

\section{Seguimento}

Todos os pacientes foram encaminhados para unidade de cuidados intensivos após o procedimento, sendo realizada tentativa de suspensão de prostaglandina endovenosa. Pacientes com necessidade de abertura prolongada do ducto arterioso ou sinais de baixa complacência ventricular direita e dessaturação persistente, apesar de valvoplastia pulmonar com sucesso, eram submetidos a shunt sistêmico-pulmonar. Ecocardiograma bidimensional com Doppler em cores era realizado imediatamente após o procedimento e seriadamente para controle do crescimento ventricular direito no seguimento ambulatorial, com periodicidade mensal nos primeiros meses de vida e semestral naqueles com boa evolução após o primeiro ano de vida.

\section{Análise estatística}

Dados clínicos e hemodinâmicos foram descritos como frequência, média e desvio padrão, mediana e valores mínimos e máximos. Utilizamos o teste qui-quadrado e teste exato de Fischer para amostras pareadas, assumindo-se valor estatisticamente significante com $\mathrm{P}<0,05$. Todos os dados obtidos foram submetidos a análise pelo software SPSS versão 19.

\section{RESULTADOS}

Os dados dos 17 pacientes portadores de atresia pulmonar com septo interventricular íntegro são apresentados na Tabela 1. A maioria era do sexo masculino $(64,7 \%)$, com idade mediana de 5 dias (1 dia a 16 dias de vida) e peso médio de 3,1 $\pm 0,6 \mathrm{~kg}$. Comunicação interatrial ou forame oval patente estavam presentes em $23,5 \%$ dos casos, e 15 pacientes $(88,2 \%)$ estavam em uso de prostaglandina intravenosa previamente ao procedimento.

Dos 17 pacientes, 14 foram submetidos a perfuração da valva pulmonar com guia rígida e 3 foram submetidos a valvotomia pulmonar com radiofrequência. Perfuração valvar com a guia foi realizada com sucesso em 71,4\% (10/14) dos casos e os 4 insucessos foram submetidos a cirurgia de Brock. O diâmetro médio final do balão utilizado foi de 7,9 $\pm 3,5 \mathrm{~mm}$. Nos 3 pacientes tratados com valvotomia pulmonar com radiofrequência, a taxa de sucesso foi de 100\%.

Três pacientes $(17,6 \%)$ apresentaram complicações menores (bradicardia responsiva a implante temporário de marcapasso, oclusão arterial aguda após punção arterial e hemopericárdico após perfuração da via de saída de ventrículo direito com radiofrequência), sendo submetidos a pericardiocentese imediata e autotransfusão com melhora imediata e posterior perfuração da valva pulmonar com sucesso.

A mortalidade hospitalar foi de 23,5\% (4/17). Um paciente de 16 dias de vida, em condições extremamente instáveis, faleceu no período pós-procedimento imediato por choque cardiogênico, após ser encaminhado a valvotomia de urgência. Outro paciente faleceu por pneumotórax espontâneo 7 dias após o procedimento. Mortalidade ocorreu em 2 pacientes (50\%) dos 4 encaminhados a cirurgia de Brock, por falha na tentativa de perfuração valvar com guia rígida.

O seguimento médio dos pacientes foi de $69 \pm$ 75,5 meses. A média do gradiente VD-AP máximo medido no seguimento tardio não diferiu da obtida 24 horas após o procedimento (Figura 2). Reintervenção ocorreu em 47,1\% (8/17) dos pacientes, todas em pacientes tratados por via percutânea: 2 pacientes foram submetidos a implante de homoenxerto em posição pulmonar; 2, a oclusão percutânea de comunicação interatrial; 1, a oclusão percutânea de persistência do canal arterial; 2, a shunt sistêmico-pulmonar; e 1 paciente foi submetido à nova valvoplastia percutânea. Um paciente apresentou síndrome de veia cava superior como complicação de cirurgia de ressecção infundibular e posicionamento de homoenxerto 2 meses após a valvotomia, falecendo 2 dias após o procedimento cirúrgico.

Os dados dos 30 pacientes portadores de estenose pulmonar crítica estão apresentados na Tabela 2. Nesse grupo, 16 neonatos eram do sexo masculino $(53,4 \%)$, com idade mediana de 12 dias (1 dia a 25 dias) e peso médio de $3 \pm 1,4 \mathrm{~kg}$. Infusão contínua de prostaglandina foi utilizada em $63 \%$ dos casos antes do procedimento. Dois pacientes eram portadores de comunicação interventricular, 1 era portador de estenose aórtica, 1 era portador de anomalia de Ebstein e 1, de estenose tricúspide.

O sucesso da valvoplastia pulmonar foi alcançado em todos os pacientes. As complicações menores relacionadas ao procedimento ocorreram em 13,3\% dos pacientes: 2 apresentaram flutter atrial e 2, reação infundibular sem instabilização hemodinâmica.

O único caso de óbito aconteceu em paciente com estenose pulmonar crítica associada a anomalia de Ebstein, por sepse, após shunt de urgência, 3 dias após a valvoplastia.

O seguimento médio dos pacientes desse grupo foi de 46,4 \pm 78,6 meses. O gradiente instantâneo máximo da valva pulmonar medido ao ecocardiograma foi de $79,5 \pm 28,5 \mathrm{mmHg}$ antes do procedimento, de $15,6 \pm 10,1 \mathrm{mmHg}$ após a valvoplastia pulmonar, e de $32,4 \pm 15,4 \mathrm{mmHg}$ no seguimento tardio (Figura 3). A taxa de reintervenção foi de 23,3\% (7/30) dos casos, com ampliação da via de saída de ventrículo direito em 2 pacientes, valvoplastia percutânea em 1 paciente, e shunt sistêmico-pulmonar em 4 pacientes. 
TABELA 1

Dados demográficos do grupo 1 (atresia pulmonar com septo interventricular íntegro)

\begin{tabular}{|c|c|c|c|c|c|c|c|c|c|c|c|}
\hline Paciente & $\begin{array}{l}\text { Idade } \\
\text { (dias) }\end{array}$ & Sexo & $\begin{array}{c}\text { Peso } \\
\text { (kg) }\end{array}$ & $\begin{array}{l}\text { Prosta- } \\
\text { glandina } \\
\text { pré }\end{array}$ & $\begin{array}{l}\text { Prosta- } \\
\text { glandina pós } \\
\text { (dias) }\end{array}$ & Valvotomia & Balão & Reintervenção & $\begin{array}{c}\text { Tempo de } \\
\text { reintervenção } \\
\text { (dias) }\end{array}$ & $\begin{array}{l}\text { Segui- } \\
\text { mento } \\
\text { (meses) }\end{array}$ & Óbito \\
\hline 1 & 9 & $\mathrm{~F}$ & 3 & Não & 1 & Guia rígida & 5 & Homoenxerto & 29 & 112 & Não \\
\hline 2 & 1 & M & 2,6 & Sim & 4 & Guia rígida & 8 & $\begin{array}{l}\text { Valvoplastia } \\
\text { percutânea }\end{array}$ & 210 & 128 & Não \\
\hline 3 & & $\mathrm{~F}$ & 3 & Sim & 15 & Guia rígida & 8 & Shunt & 15 & 108 & Não \\
\hline 4 & 8 & M & 3,2 & Sim & 8 & Guia rígida & 7 & - & - & 72 & Não \\
\hline 5 & 1 & $\mathrm{~F}$ & 3 & Sim & 0 & Guia rígida & 9 & $\begin{array}{c}\text { Patência do } \\
\text { canar arterial } \\
\text { Mola }\end{array}$ & 98 & 75 & Não \\
\hline 6 & 2 & M & 3 & Sim & 2 & Radiofrequência & 10 & $\begin{array}{c}\text { Comunicação } \\
\text { interatrial } \\
\text { Amplatzer }\end{array}$ & 1650 & 0,1 & Não \\
\hline 7 & 1 & M & 3,5 & Sim & 3 & Guia rígida & 8 & - & - & 58 & Não \\
\hline 8 & 16 & M & 3 & Sim & 1 & Guia rígida & 8 & - & - & 0,1 & Sim \\
\hline 9 & 1 & M & 3.5 & Sim & 0 & Guia rígida & 8 & $\begin{array}{c}\text { Comunicação } \\
\text { interatrial } \\
\text { Amplatzer }\end{array}$ & 35 & 39 & Não \\
\hline 10 & 2 & M & 3,3 & Sim & 1 & Guia rígida & 8 & - & - & 33 & Não \\
\hline 11 & 2 & $\mathrm{~F}$ & 3,9 & Sim & 20 & Radiofrequência & 8 & Shunt & 13 & 270 & Não \\
\hline 12 & 3 & M & 3,6 & Sim & 1 & Guia rígida & 8 & - & - & 0,23 & Sim \\
\hline 13 & 1 & $\mathrm{~F}$ & 2,9 & Sim & 6 & Radiofrequência & 7 & Homoenxerto & 72 & 2 & Sim \\
\hline 14 & 2 & M & 1,4 & Sim & 5 & - & - & $\begin{array}{l}\text { Cirurgia de } \\
\text { Brock }\end{array}$ & 5 & 0,3 & Sim \\
\hline 15 & 7 & $\mathrm{~F}$ & 3,5 & Sim & 1 & - & - & $\begin{array}{c}\text { Cirurgia de } \\
\text { Brock }\end{array}$ & 1 & 0,2 & Sim \\
\hline 16 & 8 & M & 3,5 & Sim & 1 & - & - & $\begin{array}{c}\text { Cirurgia de } \\
\text { Brock }\end{array}$ & 1 & 59 & Não \\
\hline 17 & 8 & M & 3,5 & Não & 1 & - & - & $\begin{array}{c}\text { Cirurgia de } \\
\text { Brock }\end{array}$ & 1 & 29 & Não \\
\hline
\end{tabular}

$F=$ feminino; $M=$ masculino.

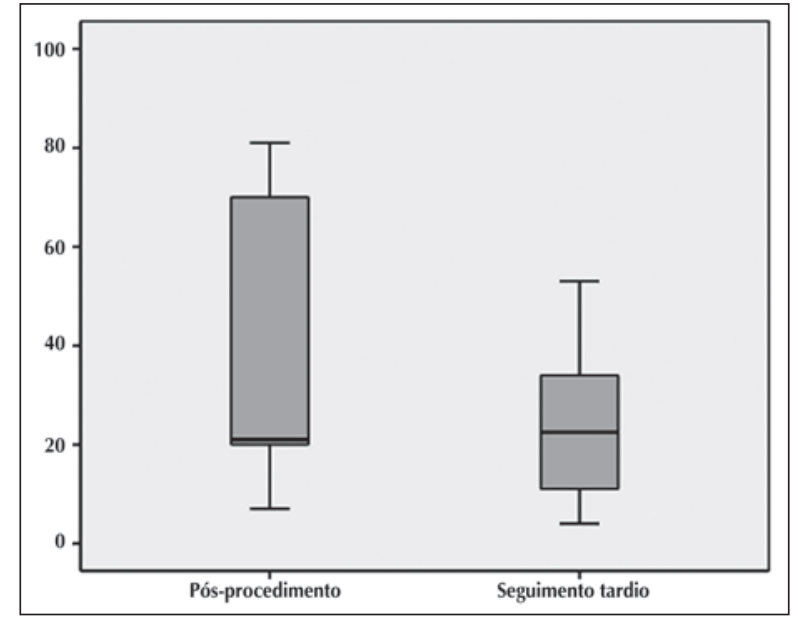

Figura 2 - Comparação entre os gradientes ventrículo direito-artéria pulmonar 24 horas após valvoplastia pulmonar e no seguimento tardio no grupo $1(P=0,46)$.

\section{DISCUSSÃO}

A estenose pulmonar e a atresia pulmonar com septo interventricular íntegro representam $10 \%$ e 2,5\%, respectivamente, das cardiopatias congênitas. ${ }^{5} \mathrm{O}$ presente estudo demonstra a efetividade do tratamento percutâneo da estenose pulmonar crítica e atresia pulmonar com septo interventricular íntegro em neonatos em um serviço terciário e sua evolução clínica a médio prazo.

As primeiras tentativas de alívio da estenose pulmonar datam de $1953^{6}$, por meio de técnicas rudimentares. A evolução ocorreu em 1979, quando Semb et al. ${ }^{7}$ relatam a técnica de valvoplastia pulmonar utlizando um cateter angiográfico tipo Berman ${ }^{\mathrm{TM}}$. Porém, o divisor de águas no tratamento percutâneo da estenose pulmonar foi realizado por Kan et al. ${ }^{2}$, com a descrição do uso de um balão desenvolvido especificamente para esse fim. Na atresia pulmonar com septo interventricular íntegro, os avanços tiveram início 
TABELA 2

Dados demográficos do grupo 2 (estenose pulmonar crítica)

\begin{tabular}{|c|c|c|c|c|c|c|c|c|c|c|}
\hline Paciente & $\begin{array}{l}\text { Idade } \\
\text { (dias) }\end{array}$ & Sexo & $\begin{array}{c}\text { Peso } \\
\text { (kg) }\end{array}$ & $\begin{array}{c}\text { Prostaglandina } \\
\text { pré }\end{array}$ & $\begin{array}{c}\text { Prostaglandina } \\
\text { pós } \\
\text { (dias) }\end{array}$ & Balão & Reintervenção & $\begin{array}{c}\text { Tempo de } \\
\text { reintervenção } \\
\text { (dias) }\end{array}$ & $\begin{array}{l}\text { Seguimento } \\
\text { (meses) }\end{array}$ & Óbito \\
\hline 1 & 11 & M & 3,1 & Não & 1 & 3 & & - & 392 & Não \\
\hline 2 & 2 & M & 2.7 & Não & 1 & 10 & & - & 138 & Não \\
\hline 3 & 15 & M & 3,5 & Não & 1 & 10 & & - & 0,1 & Não \\
\hline 4 & 3 & $\mathrm{~F}$ & 3,3 & Sim & 1 & 8 & & - & 13 & Não \\
\hline 5 & 8 & $F$ & 3 & Não & 1 & 10 & & - & 0,1 & Não \\
\hline 6 & 11 & $\mathrm{~F}$ & 3 & Não & 1 & 8 & $\begin{array}{l}\text { Valvoplastia } \\
\text { percutânea }\end{array}$ & 134 & 127 & Não \\
\hline 7 & 2 & $\mathrm{~F}$ & 2,8 & Sim & 1 & 10 & & - & 110 & Não \\
\hline 8 & 1 & $\mathrm{~F}$ & 3 & Sim & 1 & 8 & & - & 1 & Não \\
\hline 9 & 2 & M & 3,1 & Sim & 1 & 8 & & - & 135 & Não \\
\hline 10 & 3 & M & 3 & Sim & 6 & 8 & Shunt & 7 & 2 & Não \\
\hline 11 & 2 & M & 2 & Sim & 4 & 8 & Shunt & 4 & 0,1 & Não \\
\hline 12 & 25 & $\mathrm{~F}$ & 2 & Não & 1 & 7 & $\begin{array}{c}\text { Ampliação da } \\
\text { via de saída de } \\
\text { ventrículo direito }\end{array}$ & 300 & 84 & Não \\
\hline 13 & 2 & $M$ & 3 & Sim & 1 & 8 & & - & 72 & Não \\
\hline 14 & 1 & $M$ & 3 & Sim & 8 & 8 & & - & 39 & Não \\
\hline 15 & 1 & F & 3 & Sim & 1 & 6 & Shunt & 3 & 0,1 & Sim \\
\hline 16 & 1 & $M$ & 3,6 & Sim & 2 & 8 & & - & 36 & Não \\
\hline 17 & 8 & $F$ & 3,6 & Sim & 1 & 8 & & - & 48 & Não \\
\hline 18 & 8 & $\mathrm{~F}$ & 3,285 & Não & 1 & 12 & & - & 0,1 & Não \\
\hline 19 & 13 & $F$ & 2,8 & Não & - & 9 & & - & 25 & Não \\
\hline 20 & 4 & $\mathrm{~F}$ & 2,6 & Não & 1 & 8 & $\begin{array}{c}\text { Ampliação da } \\
\text { via de saída de } \\
\text { ventrículo direito }\end{array}$ & 240 & 23 & Não \\
\hline 21 & 20 & $M$ & 2,5 & Sim & 1 & 8 & & - & 41 & Não \\
\hline 22 & 17 & $F$ & 4 & Não & 1 & 8 & & - & 46 & Não \\
\hline 23 & 3 & $M$ & 3,3 & Sim & 1 & 8 & & - & 43 & Não \\
\hline 24 & 4 & $F$ & 2,9 & Sim & 1 & 8 & & - & 3 & Não \\
\hline 25 & 1 & $M$ & 2,8 & Sim & 1 & 8 & & - & 0,1 & Não \\
\hline 26 & 1 & $M$ & 3 & Sim & 3 & 9 & Shunt & 8 & 0 & Não \\
\hline 27 & 19 & $M$ & 3,2 & Sim & 1 & 8 & & - & 8 & Não \\
\hline 28 & 3 & $M$ & 4,2 & Não & 1 & 10 & & - & 2 & Não \\
\hline 29 & 1 & $F$ & 3 & Sim & 1 & 8 & & - & 3 & Não \\
\hline 30 & 3 & $M$ & 1,8 & Sim & 1 & 8 & & - & 1 & Não \\
\hline
\end{tabular}

com Qureshi et al. ${ }^{8}$ e posteriormente com Hausdorf et al. ${ }^{4}$, em 1993.

Desde então, muito grupos ao redor do mundo admitiram a técnica e a aperfeiçoaram, conferindo ao procedimento eficácia e segurança a curto, médio e longo prazos, tornando a valvoplastia pulmonar procedimento de classe I, nível de evidência A, nos portadores de estenose pulmonar crítica da valva pulmonar (estenose pulmonar, cianose e dependência de canal arterial patente ao nascimento), e de classe Ila, nível de evidência $C$, para portadores de atresia pulmonar com septo interventricular íntegro e anatomia ventricular favorável, sem circulação coronária dependente do ventrículo direito. ${ }^{9}$

Vários autores descrevem sua experiência com estenose pulmonar e atresia pulmonar com septo interventricular íntegro, analisando a segurança do procedimento e, sobretudo, sua eficácia no que se refere ao 


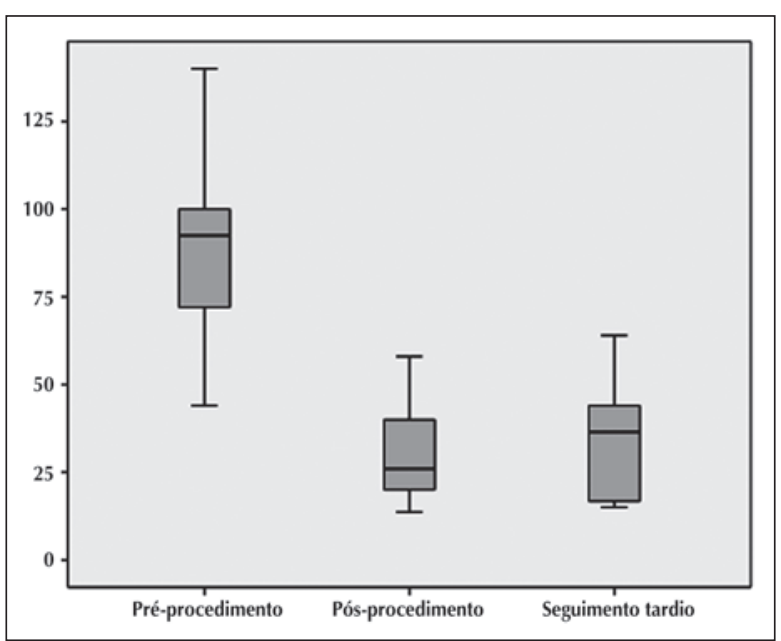

Figura 3 - Comparação entre os gradientes ventrículo direito-artéria pulmonar pré e 24 horas pós-valvoplastia $(P=0,02)$, e 24 horas pós-valvoplastia e no seguimento tardio $(P=0,73)$ no grupo 2 .

crescimento ventricular e do anel valvar pulmonar ${ }^{3}$, além do ganho, segundo alguns, do z score da valva tricúspide. ${ }^{10}$ As taxas de sucesso são cada vez maiores com menores taxas de reintervenção, principalmente com o advento de novas estratégias de valvotomia com radiofrequência. Isso se deve também ao melhor entendimento da fisiologia ventricular e à seleção de casos favoráveis à perfuração da valva pulmonar. ${ }^{11}$

As lesões residuais na maioria das vezes são leves e não obrigam a reintervenção, porém a abordagem percutânea em um segundo momento pode se fazer necessária, não para tratamento da lesão residual mas para oclusão de comunicações interatriais por cianose, canais arteriais ou shunt sistêmico-pulmonares. ${ }^{1}$

Neste estudo, a taxa de reintervenção por reestenose de $14 \%(6 / 43)$ dos pacientes é similar à descrita na literatura. ${ }^{3}$ Essa taxa foi numericamente maior nos pacientes com atresia pulmonar com septo interventricular íntegro (21,4\%) que nos pacientes com estenose pulmonar $(10 \%)$, demonstrando a maior complexidade tanto da morfologia valvar, eventualmente com hipoplasia do anel pulmonar, como da presença de estenose pulmonar subvalvar, que pode ser progressiva. ${ }^{12}$

Ainda que $14 \%$ dos pacientes tenham necessitado de shunt sistêmico-pulmonar para melhora temporária da saturação no período imediato à valvoplastia pulmonar, a ausência de pacientes submetidos a cirurgia para fisiologia univentricular evidencia a adequada seleção dos pacientes. Caspi et al. ${ }^{13}$ sugerem que a necessidade de shunt sistêmico-pulmonar esteja associada à presença de estenose infundibular; entretanto, esse dado não pôde ser avaliado no presente estudo. Dos 6 pacientes que necessitaram de prostaglandina por mais de uma semana, 4 foram submetidos a shunt sistêmico-pulmonar por fisiologia canal-dependente, a despeito da valvoplastia com sucesso. Um paciente em estado grave, com diagnóstico de anomalia de Ebstein associada, foi submetido a shunt de urgência 3 dias após o procedimento e faleceu por sepse. Tal associação assim como falência ventricular direita são descritas por Hirata et al. ${ }^{14}$ como fatores de risco para a mortalidade hospitalar. O outro paciente foi submetido a shunt 4 dias após o procedimento por dessaturação persistente e sinais significativos de baixa complacência ventricular direita.

Semelhante ao descrito previamente na literatura, o aparecimento de cianose crônica por shunt direitaesquerda por meio de comunicação interatrial ou forame oval patente em pacientes portadores de estenose pulmonar crítica não parece ser relevante, haja vista a ausência de casos de atriosseptoplastia nesse grupo de pacientes. ${ }^{13,15,16}$ Por outro lado, entre os pacientes portadores de atresia pulmonar com septo interventricular íntegro, neste estudo, 15\% necessitaram de oclusão percutânea de comunicação interatrial por cianose crônica durante o seguimento clinico. Esse dado é condizente com a literatura, encontrando-se incidência de $10 \%$ em estudo de Alwi et al. ${ }^{11}$

A taxa de mortalidade hospitalar dos pacientes de $10,6 \%(5 / 47)$ é similar à descrita na literatura ${ }^{10,14,16,17}$ e evidencia a gravidade da doença mesmo com adequada seleção de pacientes. Em se tratando apenas de casos de atresia pulmonar com septo interventricular íntegro, a mortalidade sobe para 23,5\% (4/17). Alwi et al. ${ }^{11}$, em um estudo comparando dados coletados em portadores de atresia pulmonar com septo interventricular íntegro submetidos a valvotomia por radiofrequência e valvotomia cirúrgica complementada com shunt sistêmico-pulmonar, evidenciaram taxa de mortalidade de $15 \%$ para o grupo da abordagem percutânea e de $42 \%$ para aqueles submetidos a cirurgia como tratamento inicial. Já Caspi et al. ${ }^{13}$ relatam mortalidade cirúrgica em torno de 25\%. É notável a diferença de mortalidade nos pacientes portadores de atresia pulmonar com septo interventricular íntegro (23,5\%), comparativamente à de pacientes com estenose pulmonar crítica $(3,3 \%)$, o que confirma, como já previamente comentado, que a atresia pulmonar com septo interventricular íntegro é uma afecção mais complexa que a estenose pulmonar crítica isolada.

\section{CONCLUSÕES}

A valvoplastia pulmonar na estenose pulmonar e na atresia pulmonar com septo interventricular íntegro apresenta resultados clínicos e hemodinâmicos aceitáveis, desde que se observem características anatômicas favoráveis e se mantenha a patência do fluxo pulmonar até o procedimento. A morbidade e a mortalidade dos portadores de estenose pulmonar crítica são mais baixas que as de portadores de atresia pulmonar com septo interventricular íntegro. 


\section{CONFLITO DE INTERESSES}

Os autores declaram não haver conflito de interesses relacionado a este manuscrito.

\section{REFERÊNCIAS}

1. Nugent A, Menahem S, Goh T, Butt W. Device closure of an atrial septal defect following successful balloon valvuloplasty in a neonate with critical pulmonary valve stenosis and persistent cyanosis. Pediatr Cardiol. 2000;21(2):170-1.

2. Kan J, White Jr R, Mitchell S, Gardner T. Percutaneous balloon valvuloplasty: a new method for treating congenital pulmonary valve stenosis. N Engl J Med. 1982;307(9):540-2.

3. Gildein H, Kleinert S, Goh T, Wilkinson J. Pulmonary valve annulus grows after balloon dilatation of neonatal critical pulmonary valve stenosis. Am Heart J. 1998;136(2):276-80.

4. Hausdorf G, Schneider M, Fink C. Interventional right ventricular outflow tract creation in pulmonary atresia using radiofrequency. Cardiol Young. 1993;3 Suppl:6.

5. Costa F, Kajita L, Martinez Filho E. Intervenções percutâneas em cardiopatias congênitas. Arq Bras Cardiol. 2002;78(6): 608-17.

6. Rubio A, Limon L, Soni J. Valvulotomias intracardiacas por medio de un cateter. Arch Inst Cardiol Méx. 1953;23(1):83-92.

7. Semb B, Tjonneland S, Stake G, Aabyholm G. Balloon valvotomy of congenital pulmonary valve stenosis with tricuspid insufficiency. Cardiosvasc Radiol. 1979;2(4):239-41.

8. Qureshi A, Rosenthal E, Tynan M, Anjos R, Baker E. Transcatheter laser-assisted ballon pulmonary valve dilation in pulmonic valve atresia. Am J Cardiol. 1991;67(3): 428-31.

9. Feltes TF, Bacha E, Beekman RH $3^{\text {rd }}$, Cheatham JP, Feinstein JA, Gomes AS, et al. Indications for cardiac catheterization and intervention in pediatric cardiac disease: a scientific statement from the American Heart Association. Circulation. 2011;123(22):2607-52.

10. Humpl T, Soderberg B, McCrindle B, Nykanen D, Freedom R, Williams WG, et al. Percutaneous balloon valvotomy in pulmonary atresia with intact ventricular septum. Circulation. 2003;108(7):826-32.

11. Alwi M, Geetha K, Bilkis AA, Lim MK, Hasri S, Haifa AL, et al. Pulmonary atresia with intact ventricular septum percutaneous radiofrequency-assisted valvotomy and balloon dilatation versus surgical valvotomy and Blalock Taussig shunt. J Am Coll Cardiol. 2000;35(2):468-76.

12. Alwi M. Management algorithm in pulmonary atresia with intact ventricular septum. Catheter Cardiovasc Interv. 2006 67(5):679-86

13. Caspi J, Coles J, Benson L, Freedom RM, Burrows PE, Smallhorn JF, et al. Management of neonatal critical pulmonic stenosis in the balloon valvotomy era. An Thorac Surg. 1990; 49(2):273-8

14. Hirata Y, Chen J, Quaegebeur J, Hellenbrand W, Mosca R. Pulmonary atresia with intact ventricular septum: limitations of catheter-based intervention. Ann Thorac Surg. 2007;84(2): 574-80.

15. Fedderly RT, Lloyd TR, Mendelsohn AM, Beekman RH. Determinants of successful balloon valvotomy in infants with critical pulmonary stenosis or membranous pulmonary atresia with intact ventricular septum. J Am Coll Cardiol. 1995;25(2): 460-5.

16. Tabatabaei H, Boutin C, Nykanen DG, Freedom RM, Bensosn $\mathrm{LN}$. Morphologic and hemodynamic consequences after percutaneous balloon valvotomy for neonatal pulmonary stenosis: medium-term follow-up. J Am Coll Cardiol. 1996; 27(2):473-8

17. Bartolomé FB, Fernández-Bernal CS, Feced VT. Valvulotomía percutánea de la estenosis pulmonary crítica neonatal: resultados y seguimiento a medio plazo. Rev Esp Cardiol. 1999;52: 666-70. 